\title{
51.
}

\section{SUR UN DÉTERMINANT SYMÉTRIQUE QUI COMPREND COMME CAS PARTICULIER LA PREMIÈRE PARTIE DE L'ÉQUATION SÉCULAIRE.}

\section{[Crelle's Journal für die reine und angewandte Mathematik, LXxxviII. (1880), pp. 6-9.]}

LA théorie de l'équation séculaire s'étend aisément à un déterminant symétrique beaucoup plus général. Dans le théorème de Sturm ou dans le théorème plus complet sur les intercalations que j'ai donné dans mon mémoire "Sur les rapports syzygétiques, etc.", inséré aux Philosophical Transactions*, on considère une suite de fonctions telles que trois fonctions consécutives quelconques $P, Q, R$ soient liées par l'équation

$$
P=Q^{\prime} Q-R \text {. }
$$

Dans le cas présent je m'occuperai de même d'une suite de fonctions telles qu'entre trois fonctions consécutives quelconques $P, Q, R$ on ait l'équation

$$
P R=Q^{\prime} Q-Q^{\prime \prime 2},
$$

équation qui présente avec la première cette circonstance commune que pour $Q=0$ le produit $P R$ est négatif.

Soit $D$ un déterminant symétrique quelconque dont les éléments sont des fonctions rationnelles et entières de $\lambda$, et désignons par $a, b, c, \ldots l$ les termes constants, c. à d. indépendants de $\lambda$, des éléments situés dans la diagonale de symétrie et rangés dans un ordre quelconque. Formons, comme je l'ai fait dans la preuve instantanée [p. 451 above], la suite

$$
D, \delta_{a} D, \delta_{b} \delta_{a} D, \ldots \delta_{l} \delta_{k} \ldots \delta_{a} D
$$

et soient $p, q, r$ trois quantités consécutives prises dans la série $a, b, \ldots k, l$; cela posé, on aura

$$
\left(\delta_{r} \delta_{q} \delta_{p} \ldots \delta_{a}\right) D .\left(\delta_{p} \ldots \delta_{a}\right) D=\left(\delta_{r} \delta_{p} \ldots \delta_{a}\right) D .\left(\delta_{q} \delta_{p} \ldots \delta_{a}\right) D-M^{2},
$$

$M$ étant une fonction entière de $\lambda$. La loi du signe contraire de deux termes, voisins d'un terme qui s'évanouit, de la suite considérée ci-dessus ne subit donc aucun changement.

[* Vol. I. of this Reprint, p. 545.] 
La méthode dont on s'est servi dans la preuve instantanée conduit par conséquent à ce résultat que l'entrelacement de $D$ par $\delta_{a} D$ entre des limites quelconques $p$ et $q$ est égal à la valeur numérique absolue de la différence entre le nombre des permanences de signe que présente la suite considérée ci-dessus pour les deux valeurs $\lambda=p$ et $\lambda=q$.

Je dis de plus que l'entrelacement de $D$ par $\delta_{a} D$ est égal à l'entrelacement de $D$ par $\delta_{b} D, a$ et $b$ étant deux quelconques des quantités $a, b, \ldots l$.

En effet le premier de ces deux nombres dépend uniquement du rapport des signes de $D$ et de $\delta_{a} D$ dans le voisinage des valeurs de $\lambda$ pour lesquelles $D$ s'évanouit, et pour le second de ces deux nombres on n'a qu'à substituer $\delta_{b} D$ au lieu de $\delta_{a} D$. Mais on sait que le produit $\delta_{a} D . \delta_{b} D$ excède le produit $D .\left(\delta_{a} \delta_{b}\right) D$ d'un carré positif. Les deux quantités $\delta_{a} D$ et $\delta_{b} D$ sont donc du même signe dans le voisinage des valeurs de $\lambda$ pour lesquelles $D=0$, ce qui prouve l'assertion qu'il s'agissait de démontrer.

En se bornant pour plus de simplicité au cas des entrelacements absolus, c. à $d$. au cas où $-\infty$ et $+\infty$ sont les limites de $\lambda$, on en tire le théorème suivant:

Soient

les degrés et

$$
\theta, \theta_{1}, \theta_{2}, \ldots \theta_{n}
$$$$
\mu, \mu_{1}, \mu_{2}, \ldots \mu_{n}
$$

les coefficients des plus hautes puissances de $\lambda$ dans

$$
D, \delta_{a} D,\left(\delta_{b} \delta_{a}\right) D, \ldots\left(\delta_{l} \ldots \delta_{b} \delta_{a}\right) D ;
$$

cela posé, l'entrelacement de $D$ par une quelconque des quantités $\delta_{a} D$, $\delta_{b} D, \ldots \delta_{l} D$ est la valeur numérique de la différence entre le nombre des permanences de signes que présentent les deux suites

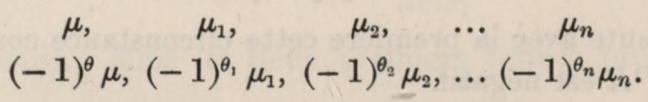

Considérons le cas particulier dans lequel les éléments du déterminant $D$ sont des fonctions linéaires de $\lambda$. Supposons de plus que tous les coefficients $\mu, \mu_{1}, \mu_{2}, \ldots \mu_{n}$ ont le signe positif. Dans ce cas l'entrelacement de $D$ par l'une quelconque des déterminants dérivés $\delta_{a} D, \delta_{b} D, \ldots \delta_{l} D$ est égal à $n$, et par conséquent toutes les racines de l'équation $D=0$ sont réelles, résultat que l'on verifie aisément.

En effet, soit $D_{1}$ le déterminant formé des parties constantes des éléments de $D$ ou, ce qui est la même chose, la valeur de $D$ pour $\lambda=0$, soit de plus $\Delta$ le déterminant formé des coefficients de $\lambda$ des éléments de $D$ ou, ce qui est la même chose, le coefficient de $\lambda^{n}$ dans le développement de $D$; cela posé, $D$ peut être regardé comme l'invariant de la forme quadratique à $n$ variables $\Phi+\lambda \Omega$, $\Phi$ et $\Omega$ étant des formes quadratiques dont les invariants sont $D_{1}$ et $\Delta$. Représentons d'une manière symbolique par

$$
(\alpha x+\beta y+\gamma z+\ldots+\lambda u)^{2}
$$


la forme quadratique $\Omega$, et par

le déterminant

$$
\alpha, \beta, \gamma, \ldots \lambda * p, q, r, \ldots t
$$

posons de plus

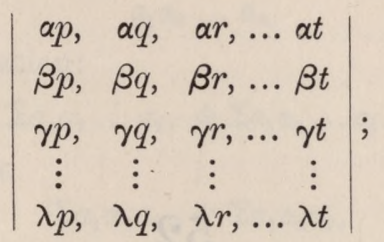

$$
\begin{array}{lrrr}
\xi= & \alpha *(\alpha x+\beta y+\gamma z+\ldots+\lambda u) \\
\eta= & \alpha \beta *( & \alpha \beta y+\alpha \gamma z+\ldots+\alpha \lambda u) \\
\zeta= & \alpha \beta \gamma *( & \alpha \beta \gamma z+\ldots+\alpha \beta \lambda u) \\
\vdots & & \\
v=\alpha \beta \gamma \ldots \lambda \dot{*}( & \alpha \beta \gamma \ldots \lambda u)
\end{array}
$$

et enfin

$$
\frac{1}{A}=\alpha^{2}, \quad \frac{1}{B}=\alpha^{2}(\alpha, \beta)^{2},
$$

$$
\frac{1}{C}=(\alpha, \beta)^{2}(\alpha, \beta, \gamma)^{2}, \ldots \frac{1}{L}=(\alpha, \beta, \ldots \kappa)^{2} \cdot(\alpha, \beta, \ldots \kappa, \lambda)^{2},
$$

où la notation $(\alpha, \beta, \ldots \lambda)^{2}$ désigne le déterminant

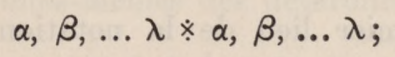

cela posé, on aura

$$
\Omega=A \xi^{2}+B \eta^{2}+\ldots+L v^{2} .
$$

Les expressions désignées d'une manière symbolique par

$$
\alpha^{2},(\alpha, \beta)^{2},(\alpha, \beta, \gamma)^{2}, \ldots(\alpha, \beta, \gamma, \ldots \lambda)^{2}
$$

étant identiques aux quantités désignées antérieurement par

$$
\mu_{n}, \mu_{n-1}, \mu_{n-2}, \ldots \mu,
$$

il est aisé de voir que l'invariant de $\Phi+\lambda \Omega$ est, à un facteur numérique près, égal à l'invariant de $\Phi_{1}+\lambda \Omega_{1}, \Phi_{1}$ représentant une fonction quadratique de fonctions linéaires à coefficients réels de $x, y, z, \ldots u$ et $\Omega_{1}$ une somme de carrés de ces mêmes fonctions linéaires.

En admettant les hypothèses faites ci-dessus on retombe donc sur le cas de l'équation séculaire.

Je me suis servi dans les calculs précédents de la notation * pour représenter une multiplication symbolique entre $\alpha, \beta, \gamma, \ldots \lambda ; \alpha^{\prime}, \beta^{\prime}, \gamma^{\prime}, \ldots \lambda^{\prime}$, tandis que je préfère garder le signe simple $\times$ pour représenter l'espèce d'opération disjonctive dont on se sert dans la théorie ordinaire de la multiplication des déterminants et qui donne naissance aux produits

$$
\left(\alpha \alpha^{\prime}, \beta \beta^{\prime}, \gamma \gamma^{\prime}, \ldots \lambda \lambda^{\prime}\right) \text {. }
$$

